\title{
Versatile Medicine? A Mini Review on the Therapeutic Approach of Chinese Medicine for Pediatric Obesity
}

\author{
C Yeunga, HY Yanga, LH Zhaoa* and XL Tonga* \\ Department of Molecular Biology Lab and Endocrinology, Guang'anmen Hospital China Academy of Chinese Medical Sciences, China
}

Submission: November 30, 2019; Published: December 16, 2019

*Corresponding author: LinHua Zhao, Department of Molecular Biology Lab and Endocrinology, Guang'anmen Hospital, Academy of Chinese Medical Sciences, Beijing, China

XiaoLin Tong, Guang'anmen Hospital, Academy of Chinese Medical Sciences, Beijing, China

\section{Abstract}

Given the unsatisfactory control on substantially increasing prevalence of pediatric obesity, multifarious therapeutic approach of Chinese medicine may open new possibility on the management of the disease. We therefore tried to briefly review the possible interventions in a manner of evidenced- based. Even reviewed RCTs showed ideal results, further study is suggested for assessment. Advocating most effort on the evaluation of Chinese medicine while using modern scientific method, exploring the Chinese medicine methodology itself is urgently needed in the meantime.

Keywords: Pediatric obesity; Chinese medicine

\section{Introduction}

The prevalence of overweight and obesity in 2014 was $19.4 \%$ (41608/214354) among age 7 to 18 Chinese children. An annual increase rate of overweight ranging from $0.27 \%$ to $0.63 \%$ and $0.1 \%$ to $0.58 \%$ of obesity [1]. This increasing trend was not observed only in china but globally. In such circumstance, also with the worry of later onset of comorbidities of childhood obesity, a series of measures had been taken place worldwide [2-5]. Especially variety of guidelines issued. Treatment recommendations from those guidelines are basically against medication approach to obesity of children and adolescents [5]. They emphasis on the lifestyle and environmental modifications. In fact, it's even hard for adult to persist on a continuous diet control and exercising, not to mention the compliance of children. In that case, the holistic concept of traditional Chinese medicine on therapeutic and heath cultivation may be a way of management of obesity of youngsters. But Chinese medicine is always questioned by its lack of evidences. Therefore, the following review is focusing on the current evidenced based researches to briefly discuss the use of Chinese medicine as the therapeutic intervention on pediatric obesity.

\section{RCTs of CM on pediatric obesity}

Clinical trials of Chinese medicine for children obesity is less than in adult. Qualified RCTs is even rare. Most of them are case studies or observational study to discuss expert experience, but without standardized reporting skill. Trials are either without randomization or without blindness. No placebo was used in those studies, diet control and exercise therapy adopted for the control group instead. No long-term follow up was mentioned. Six published RCTs included in this review, four of them are graduation thesis. All of them have shown good efficacy and safety using Chinese medicine as the intervention. Treatment methodology of Chinese medicine beneath them are either "disease differentiation" of "syndrome differentiation". Two of the studies are sorely pharmaceutical research. Two research combine herbal medication with acupuncture. One research is about Tuina therapy company by acupuncture. The last one is about partial massaging.

\section{Pharmacotherapy of CM}

One research was studied the "Wendan decoction" (fundamental herbal included: Alisma plantago-aquatica Linn, 
Poria cocos (Schw.) Wolf, Pinellia ternat, etc.) which shown a result of lowering TG, TC, LDL, apoB level after treatment and a $9.26 \%$ rebound rate in 6 months follow up. In this study a mild adverse reaction (rash) had reported and it was self-cure the second day of the study period. Herbal components in the treatment has small variation according to the different symptoms of individual under the treatment principle of "syndrome differentiation". Which imperceptibly divided the experimental group into subgroup [7]. On the contrary, the other research used fixed herbal component ("Can Fu Dao Tan" granule: Atractylodes lancea(Thunb.)DC., Cyperus rotundus L., Pinellia ternate) for a specific philegm dampness type of obesity according to syndrome differentiation. Its total efficiency of treatment group is $86.67 \%(\mathrm{P}<0.01)$, rebound rate $19.2 \%$, lower body mass index [8].

\section{Acupuncture and Tuina Therapy}

A research combined medication treatment with electroacupuncture also shown good therapeutic effect with lower BMI, leptin levels, serum TC and TG content. It used fixed component throughout the whole study (Pinellia ternate, glycyrrhiza uralensis Fisch, Poria cocos (Schw.) Wolf). This medical application is more likely under a logic of "disease syndrome", although not clearly mentioned in the article [9]. On the other hand, a study about electro-acupuncture compared with electro acupuncture company with herbal medicine. Again, acupoint and herbs selected in the treatment group is according to the individual under syndrome differentiation. Result revealed that combination treatment is better than acupuncture alone [10].

The study about Tuina therapy is no doubt followed syndrome differentiation, acupoint vary with syndrome manifestation. It made comparison to acupuncture therapy and result shown a similar therapeutic effect. The total effective rate of treatment group is $96.67 \%$, control group is $90 \%$ [11]. Last but not least, the partial massaging study, no syndrome subgrouping, carry out massage specific in hips and thighs accompany with exercise therapy. Result of study showed that exercise combine with massage was a utility way to increase the fat mobilization rate, the decrease of hip and thigh circumference became obviously keeping pace with waistline and enhanced the effect of weight loss. It also suggested 4 weeks moderate-intensity exercise is a utility way to bring down fasting insulin, improving fasting blood glucose and blood lipid [12].

\section{Discussion}

Chinese medicine has numerous therapeutic approaches toward diseases. There is cupping, moxibustion, ear acupuncture with Vaccaria segetali (Neck.) Garcke, etc. Some of them are nonintrusive. A VBM-DARTEL study revealed that microstructural difference might exit in brain because if obesity, and acupuncture therapy might influence the process of brain development, leading to reorganization of cortices on the obese children [13]. Herbal preparation is multifarious. There is decoction, bolus, granules, etc. Various combination of herbs endowed Chinese medicine with a multi targeting therapeutic approach. For example, Wen-Dan decoction mentioned above has quantified with 19 compounds by using a UHPLC-QQQ-MS/MS method [14]. Experiment also shown that Wen-Dan decoction could lower serum TC, TG, LDL level [14]. When intervention on child obesity is rather limited, Chinese medicine seems to be an option. Unfortunately, this diversity has been criticized by the majority of modern medicine as nonevidence based. Due to the insufficient and unqualified RCTs, it's been considered as expert experience and ranked at the bottom of the evidence-based pyramid. But does a RCTs or meta-analysis can truly reveal the nature and beneficial of Chinese medicine? From the above review, both syndrome and disease differentiation show good efficacy on the disease. So, what is the significance of "differentiation"? As be in the field of Chinese medicine, further study of Chinese medicine itself is warranted. Western medicine emphasis on standardized and normalized procedure, operate by quantification, paying attention on physical data. Chinese medicine navigates by metaphysics, emphasize on etiology exploration and analysis of pathogenesis, holistic concept governs entirely. Two medical system vary in every regard, how different they are is how they been valued. These precious differences may be the guidance of long-term follow-up on childhood obesity and transition to adult care.

\section{Conclusion}

To some extent, therapeutic approach of Chinese medicine on pediatric obesity has good efficacy and safe to use according to the positive outcome of the above RCTs. There is no meta-analysis on this discussion yet for further evaluation. As many would conclude Chinese medicine might use as a complementary and alternative therapeutic approach but further evidenced based trials are warranted.

\section{References}

1. Wang S, Dong YH, Wang ZH, Zou ZY, Ma J (2017) Trends in overweight and obesity among Chinese children of 7-18 years old during 19852014. Chin J Prev Med 51(4): 300-305.

2. World Health Organization. Prioritizing areas for action in the field of population-based prevention of childhood obesity: a set of tools for Member Stated to determine and identify priority areas for action.

3. World Health Organization. Population-based approaches to childhood obesity prevention.

4. Grossman DC, Bibbins-Domingo K, Curry SJ, Barry MJ, Davidson KW, et al. (2017) Screening for obesity in children and adolescents: US preventive services task force recommendation statement. JAMA 317(23): 2417-2426.

5. Styne DM, Arslanian SA, Connor EL, Farooqi IS, Murad MH, et al. (2017) Pediatric obesity-assessment, treatment, and prevention: an Endocrine Society clinical practice guideline. J Clin Endocrinol Metab 102(3): 709-57. 
6. Yang F, Miu H, Huang R (2018) Study of Wen Decoction on Liquid Metabolism and Body Mass of children with Simple Obesity . Journal of Huan University of Chinese Medicine 4: 470-474.

7. Qin Xiu (2016) Clinical observation on CanFu DaoTan decoction in treating children with simple obesity of phlegm and dampness type[D]. Heilongjiang University of Chinese Medicine.

8. Xiong JS, Hu T (2014) Clinical Research on Combined Traditiona Chinese and Western Medicine Treatment of Simple Obesity in children [J]. China Journal of Chinese Medicine 7: 1059-1061.

9. Xing Heng (2009) Traditional Chinese Medicine Integrated Treatment of Children with Simple Obesity and Leptin in Serum Clinical Observation[D]. HuBei University of Chinese Medicine.

10. Qiao Qiao (2009) Clinical Observation on Children Simple Obesity Treated by Tuina Therapy on Meridians and Acupoints [D]. Shandong University of Traditional Chinese Medicine.
11. Guo Y (2011) Research on Weight Loss Effect by Exercise Combined with massage on severe Obese Adolescents. Shanghai University of Sport.

12. Luo X J, Zhang H, Peng Y, Liu ZX, Li SL, et al. (2013) Neural mechanism study of acupuncture therapy for obese adolescence with VBM-DARTEL approach. Med Imaging 3: 350-354.

13. Bing Zhang, Dongli Qi, Xiuping Deng, Zhe Ma, Yumei Wu, et al. (2019) Quantification of Nineteen Bioactive Componenets in the Ancient Classical Chinese Medicine Formula of Wen-Den Decoction and Its commercial Preparations by UHPLC-QQQ-MS/MS[J]. Molecules 24(11): 2031.

14. Yao FY, Shi Q, Wang BZ, ZuoYM, Sun YZ (2009) Experimental research on the anti- nutritional obesity of rats with Wen-Dan Decoction [J]. Journal of Sichuan of Traditional Chinese Medicine 8: 13-15.

\section{Your next submission with Juniper Publishers} will reach you the below assets

- Quality Editorial service

- Swift Peer Review

- Reprints availability

- E-prints Service

- Manuscript Podcast for convenient understanding

- Global attainment for your research

- Manuscript accessibility in different formats

( Pdf, E-pub, Full Text, Audio)

- Unceasing customer service

Track the below URL for one-step submission https://juniperpublishers.com/online-submission.php 